\title{
Parâmetros bioquímicos clínicos de vacas de alta e média produção de leite, criadas em sistema freestall
}

\section{Clinical and biochemical parameters of high and medium milk yield cows reared in a freestall system}

\author{
Josiane Oliveira Feijó, ${ }^{* *}$ Patrícia Mattei, ${ }^{* *}$ Aline Marangon Oliveira, ${ }^{* * *}$ Carolina Bespalhok Jacometo, ${ }^{* * * *}$ \\ Vinícius Coitinho Tabeleão, ${ }^{* * * *}$ Rubens Alves Pereira, ${ }^{* *}$ Eduardo Schmitt, ${ }^{* *}$ Francisco Augusto Burkert Del Pino, ${ }^{* *}$ \\ Marcio Nunes Corrêa**
}

\begin{abstract}
Resumo
O objetivo deste trabalho foi avaliar os parâmetros bioquímico-clínicos de vacas primíparas e multíparas de alta e média produção de leite criadas em sistema freestall. Foram utilizadas 174 vacas da raça Holandês, divididas em quatro grupos: primíparas de alta produção ( $P A, n=37 ; 42,92 \pm 0,78 \mathrm{~kg}$ leite/dia); primíparas de média produção ( $\mathrm{PM}, \mathrm{n}=50 ; 26,44 \pm 0,91 \mathrm{~kg}$ leite/dia); multíparas de alta

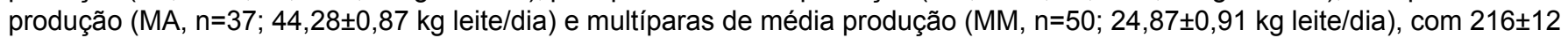
dias em lactação. Analisaram-se os seguintes metabólitos: concentrações de: colesterol total, colesterol HDL (HDL), triglicerídeos (TAG), beta-hidroxibutirato (BHB), proteínas totais (PT), albumina, ureia, e a atividade da enzima aspartato aminotransferase (AST). O grupo PM apresentou maiores concentrações de albumina $(P=0,001)$, colesterol $(P=0,001), \mathrm{HDL}(P=0,002)$ e TAG $(P=0,010)$ quando comparado com as do grupo MM. O grupo PA apresentou maiores concentrações de $\mathrm{HDL}(P=0,001)$ quando comparado com a MA. PA apresentou maiores concentrações de HDL $(P=0,023)$ e AST $(P=0,05)$ ao se comparar com PM. MA apresentou maiores concentrações de albumina $(P=0,009)$, colesterol $(P=0,041)$, HDL $(P=0,053)$ e TAG $(P=0,052)$ quando comparado com a MM. As diferenças encontradas nos metabólitos analisados tornam-se importantes no estabelecimento dos valores de referência para uma população regional de categorias semelhantes, além da possibilidade de utilização de parâmetros bioquímico-clínicos na prevenção e monitoramento de transtornos metabólicos.
\end{abstract}

Palavras-chave: lactação, marcadores metabólicos, sangue, sistema intensivo, valores de referência.

\begin{abstract}
The aim of this study was to evaluate the clinical and biochemical parameters of primiparous and multiparous cows of high and medium milk yield reared in a freestall system. One hundred seventy-four Holstein cows were used, divided into four groups: high yield primiparous (PA, $\mathrm{n}=37 ; 42.92 \pm 0.78 \mathrm{~kg}$ milk / day); medium yield primiparous (PM, $\mathrm{n}=50 ; 26.44 \pm 0.91 \mathrm{~kg}$ milk / day); high yield multiparous (MA, $n=37 ; 44.28 \pm 0.87 \mathrm{~kg}$ milk / day) and medium yield multiparous (MM, $\mathrm{n}=50 ; 24.87 \pm 0.91 \mathrm{~kg}$ milk / day) with $216 \pm 12$ days in lactation. Blood analysis included: total cholesterol, HDL cholesterol (HDL), triglycerides (TAG), betahydroxybutyrate (BHB), total protein (PT), albumin, urea, and aspartate aminotransferase (AST). The PM group had higher albumin concentrations $(P=0.001)$, cholesterol $(P=0.001)$, HDL $(P=0.002)$ and TAG $(P=0.010)$ compared to the MM group. PA group had higher HDL concentrations $(P=0.001)$ than MA. PA had higher HDL $(P=0.023)$ and AST concentrations $(P=0.05)$ compared to PM. MA had higher albumin $(P=0.009)$, cholesterol $(P=0.041), \mathrm{HDL}(P=0.053)$ and TAG $(P=0.052)$ concentrations compared to $\mathrm{MM}$. The results found in the analyzed metabolites are important in establishing reference values for a regional population, and also allow the use of these biochemical parameters in the prevention and monitoring of metabolic disorders.
\end{abstract}

Keywords: blood, intensive system, lactation, metabolic markers, references values.

\section{Introdução}

A pecuária leiteira é um dos setores de maior importância no cenário agropecuário e, consequentemente, da economia nacional brasileira. A produção leiteira em 2014 foi de 35,17 bilhões de litros (IBGE 2014), firmando o Brasil na quinta colocação no ranking mundial de produção de leite. Com aumento produtivo, há a necessidade da adoção de alternativas que maximizem a rentabilidade do rebanho, como a implantação de sistemas intensivos de produção, em que há o maior controle das condições ambientais e da dieta, proporcionando uma melhora no status de saúde e dos parâmetros produtivos (Collier et al., 2014).

\footnotetext{
${ }^{*}$ Recebido em 30 de junho de 2015 e aceito em 8 de fevereiro de 2017.

${ }^{*}$ Universidade Federal de Pelotas, Faculdade de Veterinária, Departamento de Clínica Veterinária. Pelotas, Rio Grande do Sul, Brasil. E-mail para correspondência: josianeofeijo@gmail.com

***Universidade Estadual de Londrina, Centro de Ciências Agrárias, Hospital Veterinário. Londrina, Paraná, Brasil. E-mail para correspondência: alinemarangonvet@gmail.com

****Programa de Zootecnia, Facultad de Ciencias Agropecuarias, Universidad de La Salle, Bogotá DC, Colômbia. E-mail para correspondência: cbjacometo@ gmail.com

*****Universidade do Sul de Santa Catarina, Medicina Veterinária,Tubarão, Santa Catarina, Brasil. E-mail para correspondência: vinicius.tabeleao@gmail.com
} 
Simultâneo ao aumento da produção leiteira, há um crescimento na ocorrência de transtornos metabólicos (Chapinal et al., 2011), verificando-se com maior frequência no período do periparto, compreendido entre três semanas pré-parto e três pós-parto. Neste momento ocorrem importantes alterações endócrinas, metabólicas e nutricionais que exigem do organismo uma alta capacidade de adaptação (Drackley, 1999; Chapinal et al., 2011). Durante o periparto a ingestão de matéria seca (IMS) pode ser reduzida em até $30 \%$ (Hayirli et al., 2002), e passa a ser insuficiente para suprir os requerimentos basais da vaca e do feto, causando um desequilíbrio energético conhecido como balanço energético negativo (BEN) (Esposito et al., 2014).

Em vacas de alta e média produção leiteira o BEN é mais acentuado, levando a um estado de imunossupressão, que predispõe o animal a doenças como deslocamento de abomaso, hipocalcemia, mastite, cetose, endometrite e retenção de placenta (Goff e Horst, 1997; Ingvartsen et al., 2006; Leblanc et al., 2006; Chapinal et al., 2011; Esposito et al., 2014).

Diante desta problemática, a avaliação do perfil bioquímicoclínico dos animais torna-se uma ferramenta valiosa para monitorar a condição metabólica e nutricional do rebanho (González e Silva, 2006). O balanço energético de ruminantes pode ser avaliado através das concentrações séricas de colesterol, triglicerídeos (TAG), AGNE e BHB (Pogliani e Birgel, 2007) enquanto que o balanço proteico pelas concentrações de proteínas totais (PT), ureia e albumina (González e Silva, 2006).

O objetivo deste trabalho foi avaliar os parâmetros bioquímicos clínicos de vacas leiteiras primíparas e multíparas de alta e média produção criadas em sistema freestall, gerando valores bioquímicos de referência de animais com diferentes níveis de produção mantidos num sistema de criação intensiva.

\section{Material e métodos}

Todos os procedimentos experimentais foram aprovados pelo Comitê de Ética de Experimentação Animal da Universidade Federal de Pelotas (CEEA 6553).

Este estudo foi realizado em uma fazenda comercial localizada no estado de São Paulo, no Brasil, durante o mês de junho de 2012. Foram utilizadas 174 vacas da raça Holandês, divididas em quatro grupos: primíparas de alta produção $(P A, n=37)$, que no momento da coleta apresentavam produção leiteira maior que $35 \mathrm{~kg} /$ dia (média de 42,92 $\pm 0,78 \mathrm{~kg} / \mathrm{dia}$ ); primíparas de média produção ( $P M, n=50)$, que no momento da coleta apresentavam produção leiteira menor que $35 \mathrm{~kg} /$ dia (média de $26,44 \pm 0,91 \mathrm{~kg} / \mathrm{dia}$ ); multíparas de alta produção (MA, $n=$ 37), que no momento da coleta apresentavam produção leiteira maior que $35 \mathrm{~kg}$ / dia (média de 44,28 $\pm 0,87 \mathrm{~kg} /$ dia) e multíparas de média produção $(\mathrm{MM}, \mathrm{n}=50)$, que no momento da coleta apresentavam produção leiteira menor que $35 \mathrm{~kg} /$ dia (média de $24,87 \pm 0,91 \mathrm{~kg} /$ dia). O período de lactação médio de todas as vacas avaliadas foi de $216 \pm 12$ dias. O manejo sanitário ao qual os animais eram submetidos seguia as recomendações do Ministério da Agricultura, Pecuária e Abastecimento em relação às vacinações, e os tratamentos para ectoparasitas e insetos vetores eram aplicados de acordo com o protocolo da fazenda.

Todos os animais foram mantidos em sistema freestall, recebendo uma dieta total rica em concentrado de acordo com o nível de produção. Nos grupos de alta produção (MA e PA), a dieta continha 1,74 Mcal / $\mathrm{kg}$ e nos grupos de média produção (PM e MM) 1,56 Mcal / kg (Tabela 1). A ingestão de matéria seca de cada grupo era estimada diariamente de acordo com a quantidade ofertada e as sobras, e o consumo de matéria seca durante o período experimental foi semelhante entre os grupos (PA: $24 \mathrm{~kg}$ / d; PM: 22,7 kg / d; MA: 26,9 kg / d e MM: 22,7 kg / d, $P>0,05$ ).

Tabela 1: Composição nutricional das dietas ofertadas para vacas leiteiras de alta e média produção (\%)

\begin{tabular}{lcc}
\hline Ingrediente da dieta & Alta produção & Média Produção \\
\hline Silagem de milho & 33,32 & 45,81 \\
Pré-secado & 7,30 & 5,16 \\
Caroço de algodão & 7,11 & 4,52 \\
Polpa cítrica & 12,31 & 13,42 \\
Milho úmido & - & 10,67 \\
Milho seco & 17,00 & - \\
Farelo de soja & 15,30 & 12,12 \\
Água & 0,19 & 0,00 \\
Gordura protegida & 0,95 & - \\
Mix mineral e vitamínico & 6,52 & 8,30 \\
TOTAL & 100 & 100 \\
\hline
\end{tabular}

- indica ausência do ingrediente na dieta

Amostras de sangue foram coletadas da veia coccígea. Após centrifugação a $1.000 \times g$ durante 15 minutos as amostras foram criopreservadas a $-80^{\circ} \mathrm{C}$ para posterior análise. Foram analisadas as concentrações de colesterol total, colesterol HDL, TAG, PT, albumina, ureia e a atividade da enzima aspartato aminotransferase (AST) (Labtest Diagnóstica ${ }^{\circledR}$, Lagoa Santa, MG, Brasil) e BHB (Randox, Oceanside, CA) utilizando kits comerciais. Os coeficientes de variação foram inferiores a $10 \%$ em todos os ensaios.

Os resultados foram analisados pelo Programa software Prism 5 (Graph Pad Software Inc., La Jolla, CA, USA), através de ONE WAY ANOVA, com comparação entre médias de acordo com o Teste de Tukey HSD para os metabólitos (TAG, colesterol total, colesterol HDL ureia, albumina, AST, BHB e PT). Valor de $P \leq$ 0,05 foi considerado como significativo.

\section{Resultados e discussão}

Nas tabelas 2, 3, 4 e 5, são apresentadas as concentrações séricas de albumina, AST, BHB, colesterol, colesterol HDL, $P T, T A G$ e ureia das vacas referentes às diferentes categorias (primíparas e multíparas) de média produção e alta produção.

Os indicadores bioquímicos permitem estabelecer o grau de adequação metabólica ou de homeostase em um processo bioquímico do organismo (González e Silva, 2006). Entretanto, a interpretação desses indicadores, tanto aplicado a rebanhos quanto a indivíduos é complexa (Caldeira, 2007). Isso se deve aos mecanismos que controlam os níveis sanguíneos dos metabólitos e também à grande variação desses níveis em função de fatores como raça, idade, estresse, dieta, manejo, clima, estado fisiológico e nível de produção (Pogliani e Birgel, 2007; Cozzi et al., 2011). 
Tabela 2: Concentrações sanguíneas (média \pm erro padrão) dos parâmetros bioquímicos (albumina, AST, BHB, colesterol, colesterol HDL, PT, TAG e ureia) de vacas leiteiras multíparas e primíparas de média produção

\begin{tabular}{lccc}
\hline \multicolumn{1}{c}{ Metabólito } & PM & MM & Valor de P* \\
\hline Albumina (g / dL) & $3,09 \pm 0,06$ & $2,80 \pm 0,05$ & 0,001 \\
AST $(\mathrm{U} / \mathrm{L})^{1}$ & $54,58 \pm 2,30$ & $56,07 \pm 3,42$ & 0,719 \\
BHB (mEq / L) & $0,42 \pm 0,09$ & $0,45 \pm 0,06$ & 0,732 \\
Colesterol (mg / dL) & $198,40 \pm 9,58$ & $142,10 \pm 6,60$ & 0.001 \\
Colesterol HDL (mg / dL) & $99,50 \pm 3,48$ & $85,54 \pm 2,92$ & 0,002 \\
PT (g / dL) & $9,20 \pm 0,16$ & $9,08 \pm 0,15$ & 0,551 \\
TAG (mg / dL) & $14,97 \pm 1,83$ & $9,74 \pm 0,90$ & 0,010 \\
Ureia & $46,36 \pm 1,30$ & $46,15 \pm 3,36$ & 0,953 \\
\hline
\end{tabular}

${ }^{1}$ Aspartatoaminotransferase; ${ }^{2} \beta$-hidroxi-butirato, ${ }^{3}$ Triglicerídeos, ${ }^{4}$ Proteínas totais* Valor de $P<0,05$ é considerado diferença estatística.

Tabela 3: Concentrações sanguíneas (média \pm erro padrão) dos parâmetros bioquímicos (albumina, AST, BHB, colesterol, colesterol HDL, PT, TAG e ureia) de vacas leiteiras primíparas e multíparas de alta produção

\begin{tabular}{lccc}
\hline \multicolumn{1}{c}{ Metabólito } & PA & MA & Valor de P* \\
\hline Albumina (g / dL) & $3,05 \pm 0,05$ & $3,04 \pm 0,06$ & 0,899 \\
AST $(\mathrm{U} / \mathrm{L})^{1}$ & $61,27 \pm 2,54$ & $59,41 \pm 3,91$ & 0,698 \\
BHB (mEq / L) & $0,42 \pm 0,044$ & $0,44 \pm 0,03$ & 0,795 \\
Colesterol (mg / dL) & $187,4 \pm 9,57$ & $167,7 \pm 10,83$ & 0,178 \\
Colesterol HDL (mg / dL) & $110,5 \pm 3,04$ & $93,83 \pm 3,44$ & 0,001 \\
PT (g / dL) & $8,57 \pm 0,15$ & $8,96 \pm 0,18$ & 0,108 \\
TAG (mg / dL) & $14,79 \pm 1,41$ & $12,38 \pm 0,87$ & 0,149 \\
Ureia (mg / dL) & $46,98 \pm 1,51$ & $48,22 \pm 2,07$ & 0,633 \\
\hline
\end{tabular}

${ }^{1}$ Aspartatoaminotransferase; ${ }^{2} \beta$-hidroxi-butirato, ${ }^{3}$ Triglicerídeos, ${ }^{4}$ Proteínas totais* Valor de $P<0,05$ é considerado diferença estatística.

Tabela 4: Concentrações sanguíneas (média \pm erro padrão) dos parâmetros bioquímicos (albumina, AST, BHB, colesterol, colesterol HDL, PT, TAG e ureia) de vacas leiteiras primíparas de alta e média produção

\begin{tabular}{lccc}
\hline \multicolumn{1}{c}{ Metabólito } & PA & PM & Valor de P* \\
\hline Albumina (g / dL) & $3,05 \pm 0,05$ & $3,09 \pm 0,06$ & 0,591 \\
AST (U / L) & $61,27 \pm 2,54$ & $54,58 \pm 2,30$ & 0,057 \\
BHB (mEq / L) & $0,42 \pm 0,044$ & $0,42 \pm 0,09$ & 0,737 \\
Colesterol (mg / dL) & $187,40 \pm 9,57$ & $198,40 \pm 9,58$ & 0,427 \\
Colesterol HDL (mg / dL) & $110,50 \pm 3,04$ & $99,50 \pm 3,48$ & 0,023 \\
PT (g / dL) & $8,57 \pm 0,15$ & $9,20 \pm 0,16$ & 0,007 \\
TAG (mg / dL) & $14,79 \pm 1,41$ & $14,97 \pm 1,83$ & 0,938 \\
Ureia (mg / dL) & $46,98 \pm 1,51$ & $46,36 \pm 1,29$ & 0,756 \\
\hline
\end{tabular}

${ }^{1}$ Aspartatoaminotransferase; ${ }^{2} \beta$-hidroxi-butirato, ${ }^{3}$ Triglicerídeos, ${ }^{4}$ Proteínas totais * Valor de $P<0,05$ é considerado diferença estatística.
Tabela 5: Concentrações sanguíneas (média \pm erro padrão) dos parâmetros bioquímicos (albumina, AST, BHB, colesterol, colesterol HDL, PT, TAG e ureia) de vacas leiteiras multíparas de alta e média produção

\begin{tabular}{lccc}
\hline \multicolumn{1}{c}{ Metabólito } & MA & MM & Valor de P* \\
\hline Albumina (g / dL) & $3,04 \pm 0,06$ & $2,80 \pm 0,05$ & 0,009 \\
AST $(\mathrm{U} / \mathrm{L})^{1}$ & $59,41 \pm 3,91$ & $56,07 \pm 3,42$ & 0,521 \\
BHB (mEq / L) & $0,44 \pm 0,03$ & $0,45 \pm 0,06$ & 0,967 \\
Colesterol (mg / dL) & $167,70 \pm 10,83$ & $142,10 \pm 6,60$ & 0,041 \\
Colesterol HDL (mg / dL) & $93,83 \pm 3,44$ & $85,54 \pm 2,92$ & 0,053 \\
PT (g / dL) ${ }^{3}$ & $8,99 \pm 0,18$ & $9,07 \pm 0,15$ & 0,756 \\
TAG (mg / dL) & $12,38 \pm 0,87$ & $9,74 \pm 0,90$ & 0,052 \\
Ureia (mg / dL) & $48,42 \pm 2,11$ & $46,40 \pm 3,36$ & 0,642 \\
\hline
\end{tabular}

${ }^{1}$ Aspartatoaminotransferase; ${ }^{2} \beta$-hidroxi-butirato, ${ }^{3}$ Triglicerídeos, ${ }^{4}$ Proteínas totais* Valor de $P<0,05$ é considerado diferença estatística.Parte inferior do formulário

Os TAG são uma fonte importante de energia e de ácidos graxos para a síntese de gordura no leite (Aeberhard et al., 2001). No presente estudo, vacas multíparas de alta produção apresentaram maiores concentrações de TAG em relação às multíparas de média produção $(P=0,052)$ (Tabela 5), decorrente da maior mobilização de energia para a alta produção de leite. Os níveis de TAG mais elevados em vacas primíparas de média produção em relação às multíparas de média produção $(P=$ 0,010 ) (Tabela 2) são decorrentes da maior exigência energética das vacas primíparas para o seu desenvolvimento e o do feto (NRC, 2001).

O colesterol desempenha importantes funções biológicas, sendo necessário à síntese dos hormônios esteroidais e dos ácidos biliares (González e Silva, 2006). Concomitante ao aumento da concentração de colesterol durante a lactação há um aumento das concentrações de lipoproteínas, especialmente das de alta densidade (HDL), principal transportadora do colesterol em bovinos (González e Silva, 2006; Pogliani e Birgel, 2007). No presente estudo, todos os animais apresentaram níveis acima dos valores fisiológicos para primíparas e multíparas, segundo os valores de referência de Pogliani e Birgel (2007), que utilizaram vacas primíparas e multíparas em lactação do estado de São Paulo (86,4-105,0 mg / dL para primíparas e 116,0-147,9 mg / dL para multíparas). Apesar dos grupos serem divididos entre alta e média produção nesta propriedade, a média de produção de leite desses animais é considerada alta, contribuindo para o aumento das concentrações de colesterol. Já em outro estudo de Cozzi e colaboradores (2011), que utilizaram vacas no início e na metade da lactação, os níveis de colesterol variaram de 112$266 \mathrm{mg} / \mathrm{dL}$ para vacas no início da lactação e entre 158-297mg/ $\mathrm{dL}$ na metade da lactação, apresentando um grande intervalo de variação para este metabólito.

Há uma carência de estudos que esclareçam os mecanismos de regulação das alterações do metabolismo do colesterol durante o ciclo da lactação em vacas. Em outras espécies animais como ratos, o início da lactação está associado com mudanças marcadas do metabolismo hepático do colesterol, com um aumento do pool do organismo, que será direcionado para a formação de ácidos biliares e para a síntese e secreção de 
lipoproteínas, utilizadas para disponibilizar colesterol e TAG para a síntese de lipídios do leite (Smith et al., 1998; Athippozhy et al., 2011). Estudos a nível molecular sugerem que os mecanismos de homeostase do colesterol em vacas durante o período de transição são similares (Schlegel et al., 2012); entretanto, não há descrição para outros períodos da lactação. Diante disso, observa-se que os níveis de colesterol são influenciados tanto pelas vacas serem primíparas ou multíparas, quanto pela produção de leite - vacas de alta produção apresentam níveis séricos maiores em relação às de média produção nos grupos das multíparas $(P=0,041)$ (Tabela 5) bem como vacas primíparas apresentam níveis maiores do que as multíparas nos grupos de média produção $(P=0,001)$ (Tabela 2), fato atrelado ao transporte de lipídios para a síntese de gordura no leite, além da síntese de hormônios esteroides, necessários para o restabelecimento da atividade reprodutiva (Aeberhard et al., 2001).

Condizente com os resultados encontrados para o colesterol, os níveis séricos de colesterol HDL foram maiores em vacas de alta produção do que de média produção, independentemente do número de lactações (PA $\times$ PM: $P=0,023$; MA $\times$ MM: $P=$ 0,053 ) (Tabelas 45 , respectivamente) e maiores em primíparas do que em multíparas, independentemente do nível de produção (PM $\times$ MM: $P=0,002 ; P A \times$ MA: $P=0,001$ ) (Tabelas 2 e 3 , respectivamente), em decorrência de sua importante função como principal carreadora do colesterol em bovinos.

As concentrações circulantes de BHB podem ser utilizadas para medir a homeorrese em relação ao BEN. Os níveis deste metabólito refletem o nível da oxidação lipídica do fígado, que quando excedida, resulta na produção excessiva de corpos cetônicos e o aumento de suas concentrações sanguíneas, podendo predispor o animal a diversas doenças de produção (Chapinal et al., 2012; Leblanc, 2010). Neste estudo, os níveis de BHB séricos foram semelhantes entre os grupos de alta e média produção, independentemente das vacas serem multíparas ou primíparas, indicando que não houve uma grande mobilização lipídica no período avaliado. A elevação dos níveis sanguíneos de BHB tem maior incidência durante a primeira semana pósparto, quando ocorre uma lipomobilização mais intensa. Além disso, este resultado pode estar relacionado com a densidade energética da ração, que impediu um BEN mais severo e culminou na manutenção do equilíbrio energético (Campos et al., 2007; Mcart et al., 2013).

As proteínas plasmáticas são constituídas principalmente pela albumina, globulinas e fibrinogênio, e o fígado é o principal órgão de síntese. Essas proteínas estão envolvidas em diversas funções, que vão desde o transporte de nutrientes até a regulação da pressão osmótica e da viscosidade e $\mathrm{pH}$ sanguíneo. A produção dessas proteínas está diretamente relacionada com o status nutricional do animal à funcionalidade hepática (González e Silva, 2006). Neste estudo não foram observadas diferenças nos níveis de proteínas totais, indicando que a dieta supria as demandas proteicas dos animais, a exemplo do estudo de Cozzi e colaboradores (2011), que avaliou vacas multíparas e primíparas e não observou diferença nos níveis deste metabólito. Outro estudo que avaliou vacas alimentadas com dieta TMR durante todo o período de lactação demonstrou que o nível sérico médio de proteínas totais durante todo o período foi $9,7 \mathrm{~g} / \mathrm{dL}$, valor semelhante ao encontrado em nosso estudo, na metade da lactação. Observou-se que não houve uma grande variação no nível proteico, confirmando este metabólito como um bom indicador dos níveis dietéticos de proteína (Nozad et al., 2013). Do mesmo modo, não foram observadas diferenças nos níveis de ureia, metabólito que reflete a relação energia:proteína da dieta (Wittwer et al., 1993). Seus níveis séricos são influenciados por uma grande variedade de parâmetros, que incluem: a ingestão de proteína na dieta e a sua degradabilidade ruminal, a ingestão de proteína em relação às necessidades e a função hepática e renal (Van Saun, 2007). Os resultados encontrados indicam que a relação de energia:proteína na dieta encontrava-se em equilíbrio, e que o aporte proteico era suficiente para suprir as demandas.

Os níveis de albumina refletem a disponibilidade de aminoácidos e seu declínio frente a uma deficiência proteica na dieta. Os valores de referência para bovinos constam com uma variação de 2,7 a 3,8g / dL (Kaneko et al., 2008). No presente estudo, todos os animais apresentaram níveis dentro dos valores fisiológicos; entretanto, foram observadas menores concentrações do metabólito no grupo MM $(2,80 \pm 0,5)$ em relação ao grupo PM $(3,09 \pm 0,06)(P=0,001)$ (Tabela 2), e menores concentrações no grupo $\mathrm{MM}(2,80 \pm 0,05)$ em relação ao grupo MA $(3,04 \pm 0,06)(P=0,009)$ (Tabela 5). Com o avanço da lactação há um aumento na demanda de aminoácidos para a síntese de proteínas do leite, reduzindo a síntese de outras proteínas e diminuindo desta forma os níveis sanguíneos de albumina (Wittwer, 2000; Caldeira, 2005).

A AST é uma enzima que possui correlação positiva com a atividade da glândula mamária, problemas hepáticos e cardíacos. Alterações na função hepática devido à infiltração gordurosa do fígado em vacas leiteiras estão associadas ao aumento das concentrações de AST (Roberts e Reid, 1993). Neste estudo, vacas primíparas de alta produção apresentaram níveis maiores de AST em relação ao grupo PM $(P=0,057)$ (Tabela 4). A atividade da AST pode ser utilizada como indicador do aumento da atividade gliconeogênica e atividade enzimática no tecido hepático, muscular e nos eritrócitos (Santos et al., 2007), condizente com a alta demanda energética para a produção de leite observada nos animais deste estudo.

\section{Conclusões}

A relação entre os indicadores do metabolismo energético e proteico e a produção leiteira precisa sempre ser avaliada a partir de um conjunto de parâmetros que representem o real status metabólico dos animais. A pouca variação observada nos marcadores bioquímicos clínicos entre vacas primíparas e multíparas de diferentes níveis de produção no terço final de lactação avaliados neste estudo é importante para que os valores de referência de uma população regional, com suas particularidades de clima, raça e sistema de criação sejam conhecidos e melhor utilizados para a correta interpretação dos perfis metabólicos. Além disso, esses marcadores podem ser utilizados como auxiliares na prevenção e monitoramento de transtornos metabólicos. 


\section{Referências}

AEBERHARD, K.; BRUCKMAIER, R.M.; BLUM, J. Metabolic, enzimatic and endocrine status in high yielding dairy cows. Part 2. Journal of Veterinary Medicine, v. 48, n. 2, p. 11-127, 2001.

ATHIPPOZHY, A.; HUANG, L.; WOOTON-KEE, C.R.; ZHAO, T.; JUNGSUWADEE, P.; STROMBERG, A.J.; VORE, M. Differential gene expression in liver and small intestine from lactating rats compared to age matched virgin controls detects increased mRNA of cholesterol biosynthetic genes. BMC Genomics, v. 12, n. 95, p.1-17, 2011.

CALDEIRA, R.M. Monitorização da adequação do plano alimentar e do estado nutricional em ovelhas. Revista Portuguesa de Ciências Veterinárias, v. 100, n. 555-556, p. 125-139, 2005.

CALDEIRA, R.M.; BELO, A.T.; SANTOS, C.C.; VAZQUES, M.I.; PORTUGAL, A. V. The effect of long-term feed restriction and over-nutrition on body condition score, blood metabolites and hormonal profiles in ewes. Small Ruminant Research, v. 68, n. 3, p. 242-255, 2007.

CAMPOS, R.; GONZÁLEZ, F.; COLDEBELLA, A.; LACERDA, L. Indicadores do metabolismo energético no pós-parto de vacas leiteiras de alta produção e sua relação com a composição do leite. Ciência Animal Brasileira, v. 8, n. 2, p. 241-249, 2007.

CHAPINAL, N.; CARSON, M.E.; DUFFIELD, T.F.; CAPEL, M.; GODDEN, S.; OVERTON, M.; SANTOS, J.E.P.; LEBLANC, S.J. The association of serum metabolites with clinical disease during the transition period. Journal of Dairy Science, v. 94, n. 10, p. 489490, 2011.

CHAPINAL, N.; CARSON, M.E.; LEBLANC, S.J.; LESLIE, K.E.; GODDEN, S.; CAPEL, M.; SANTOS, J.E.P.; OVERTON, M.W.; DUFFIELD, T.F. The association of serum metabolites in the transition period with milk production and early-lactation reproductive performance. Journal of Dairy Science, v. 95, n. 3, p.130-1309, 2012.

COLLIER, R. J.; HALL, L.W.; SMITH, J. F. Intensive livestock systems for dairy cows. In: FUHRER, J.; GREGORY, P. Climate change impact and adaptation in agricultural systems. 1ed. United Kingdom: CABI, 2014, p.110-123.

COZZI, G.; RAVAROTTO ,L.; GOTTARDO, F.; STEFANI, A.L.; CONTIERO,B.; MORO, L.; BRSCIC, M.; DALVIT, P. Short communication: Reference values for blood parameters in Holstein dairy cows: Effects of parity, stage of lactation, and season of production. Journal of Dairy Science, v. 94, n. 8, p. 3895-3901, 2011.

DRACKLEY, J.K. Biology of dairy cows during the transition period: the final frontier? Journal of Dairy Science, v. $82, \mathrm{n} .11, \mathrm{p}$. 2259-2273, 1999.

ESPOSITO, G.; PETE, C.; IRONS, P.C.; WEBB, E.C.; CHAPWANYA, A. Interactions between negative energy balance, metabolic diseases, uterine health and immune response in transition dairy cows. Animal Reproduction Science, v.144, n. 3, p. 60-71, 2014.

FAOSTAT - Food and Agriculture Organization of the United Nations: Top production - Milk, whole fresh cow - 2012. Disponível em http://faostat.fao.org/site/339/default.aspx. Acesso em 13 de abril de 2015.

GOFF, J.P.; HORST, R.L. Physiological changes at parturition and their relantioship to metabolic disorders. Journal of Dairy Science, v. 80, n. 7, p. 1260-1268, 1997.
GONZÁLEZ, F.H.D; SILVA, S.C. Introdução à bioquímica clínica veterinária. 2ed. Porto Alegre: Editora da Universidade Federal do Rio Grande do Sul, 2006, 357 p.

HAYIRLI, A.; GRUMMER, R.R.; NORDHEIM, E.V.; CRUMP, P.M. Animal and dietary factors affecting feed intake during the prefresh transition period in Holsteins. Journal of Dairy Science, v. 85, n.12, p. 3430-3443, 2002.

INSTITUTO BRASILEIRO DE GEOGRAFIA E ESTATÍSTICA IBGE Produção Pecuária Municipal - 2014. Disponível em: http:// biblioteca.ibge.gov.br/visualizacao/periodicos/84/ppm_2014_v42_ br.pdf. Acesso em 07/11/2016.

INGVARTSEN, K.L. Feeding and management related diseases in the transition cow: Physiological adaptations around calving and strategies to reduce feeding related diseases. Animal Feed Science Technology, v.126, n. 3, p.175-213, 2006.

LEBLANC, S. J.; KLISSEMORE, D.; KELTON, D.F.; DUFFIELD, T.F.; LESLIE, K.E. Major advances in disease prevention in dairy cattle. Journal of Dairy Science, v. 89, n. 4, p. 1267-1279, 2006.

LEBLANC, S. Monitoring metabolic health of dairy cattle in the transition period. Journal of Reproduction and Development, v. 56, Suppl, 2010.

KANEKO, J. J.; HARVEY, J.W.; BRUSS, M.L. Clinical biochemistry of domestic animals. 6th ed. San Diego: Academic Press, 2008, $928 \mathrm{p}$.

MCART, J.A.A.; NYDAM, D.V.; OETZEL, G.R.; OVERTON, T.R.; OSPINA, P.A. Elevated non-esterified fatty acids and $\beta$-hydroxybutyrate and their association with transition dairy cow performance. The Veterinary Journal, v. 198, n. 3, p. 560-570, 2013.

NOZAD, S.H.; RAMIN, A.G.; MOGHADAM, G.H.; ASRI-REZAEIS; KALANTARY, L.; BABAPOUR, A.; RAMIN, S.; PHILLIPS, C.J.C. Monthly and seasonal evaluation of dietary nutrients and their relationships with blood and milk parameters in lactating dairy cows. Acta Veterinaria (Beograd), v. 63, n. 2-3, p. 255-268, 2013.

NRC - Nutrient Requirements for Dairy Cattle. 7. ed. Washington: National Academy Press, 2001, 401 p.

POGLIANI, F.C.; BIRGEL, E.J. Valores de referência do lipidograma de bovinos da raça holandesa, criados no Estado de São Paulo. Brazilian Journal of Veterinary Research and Animal Science, v. 44, n. 5, p. 373-383, 2007.

ROBERTS, C.J.; REID, I.M. Fat cow syndrome and subclinical fatty liver. In: HOWARD, J.L. Current Veterinary Therapy - Food Animal Practice.3ed. Philadelphia: WB Saunders, 1993, p. 315318.

SANTOS, C.A.J.; RIET-CORREA, F.; DANTAS, A. F. M.; BARROS, S. S.; MOLYNEUX, R.J.; MEDEIROS, R. M. T.; SILVA, D. M.; OLIVEIRA, O. F. Toxic hepatopathy in sheep associated with the ingestion of the legume Tephrosiacinerea. Journal Veterinary Diagnosis Investigation, v. 19, n. 6, p. 690-694, 2007.

SCHLEGEL, G.; RINGSEIS, R.; KELLER, J.; SCHWARZ, F.J.; EDER, K. Changes in the expression of hepatic genes involved in cholesterol homeostasis in dairy cows in the transition period and at different stages of lactation. Journal of Dairy Science, v. 95, n. 7, p. 3826-3836, 2012.

SMITH, J. L.; LEAR, S.R.; FORTE, T.M.; KO, W.; MASSIMI, M.; ERICKSON, S.K. Effect of pregnancy and lactation on lipoprotein and cholesterol metabolism in the rat. Journal of Lipid Research, v. 39 , n.11, p. 2237-2249, 1998. 
VAN SAUN, R. Metabolic profiling of transition cows: can we predict impending problems? Danish Bovine Practitioner Seminar, Denmark, p. 1-8, 2007.

WITTWER, F.; OPITZ, H.; REYES, J.; CONTRERAS, P.A.; $B O ̈ H M W A L D, H$. Determinación de urea en muestras de leche de rebaños bovinos para el diagnostico de desbalance nutricional. Archivo Medico Veterinario, v. 25, n. 2, p. 165-172, 1993.
WITTWER, F. Diagnóstico dos desequilíbrios metabólicos de energia em rebanhos bovinos. In: GONZÁLES, F.H.D.; BARCELLOS, J.O.; OSPINA, H.; RIBEIRO, L.A.O. Perfil metabólico em ruminantes: seu uso em nutrição e doenças nutricionais. Porto Alegre: Editora da Universidade Federal do Rio Grande do Sul, 2000, p.09-22. 